\title{
A New Pricing Function for Power Control Game in Wireless Data Networks
}

\author{
Abdorasoul Ghasemi, Karim Faez \\ Department of Electrical Engineering \\ AmirKabir University of Technology \\ Tehran, Iran, 15914 \\ Email: \{arghasemi, kfaez\}@aut.ac.ir
}

\author{
Mehdi Dehghan \\ Department of Computer Engineering \\ AmirKabir University of Technology \\ Tehran, Iran, 15914 \\ Email: dehghan@ce.aut.ac.ir
}

\begin{abstract}
This paper presents a new pricing function for noncooperative power control game in a single cell CDMA data network. Considering a utility function for each terminal, the purpose of power control in wireless data networks is to maximize network utility. In the proposed game, the pricing function is a linear function of the terminal's Signal to Interference plus Noise Ratio (SINR). We first prove that the new game is a supermodular game and then we show the strategy space of the new game is such that it is possible to reach better equilibrium point compared to pricing function based on terminal's power. Simulation results show that the game with the proposed pricing function can improve the utility and power consumption of the terminals at equilibrium.
\end{abstract}

\section{INTRODUCTION}

Wireless networks are influenced by time varying channel due to effects such as multipath fading, shadowing, and path losses. A general strategy to combat these effects is through the dynamic allocation of resources based on the states of the user channel [1].

Transmitter power is one of these resources that need to be assigned dynamically. The purpose of Power Control (PC) algorithms is to determine each user power level to provide Quality of Service (QOS) requirements, without causing unnecessary interference for others as well as using the minimum power to extend the battery life of mobile nodes.

Current PC algorithms for wireless telephone networks are used to maximize the number of conversations that can achieve a certain QOS simultaneously. The QOS requirement for cellular telephone systems is usually indicated by a minimum Signal to Interference plus Noise Ratio (SINR), to provide acceptable subjective speech quality at the receiver [2]. Based on this requirement, efficient and distributed power control algorithms have been developed for wireless telephone networks [3], [4].

The QOS objective for data networks differs from the one for telephone networks. Despite the voice communication, which is delay sensitive and error tolerable up to a threshold, the data communication is error sensitive and delay tolerable. The QOS objective for data network users is indicated by a utility function. The Utility function is a measure of the satisfaction the user receives because of its decisions [2].

It is known that the current distributed algorithms for telephone networks are not efficient for data networks due to the natural differences in the utility functions for voice and data applications [2]. Therefore, development of new PC algorithms is required. The aim of PC algorithms for wireless data networks is to maximize the network utilization, which may be constrained by a minimum utility for each user. The network utilization is the sum of all individual users' utility.

This modeling approach for PC based on utility functions, is called economic modeling. Game theory is the analytical framework, which is used for the analysis of these models. Game theory can be used for analyzing the interaction of some decision makers with conflicting objectives. Another reason that makes the game theory an appealing tool for PC problem is that it is based on distributed solutions, which is an important factor for any PC algorithm to be scalable and easily implementable [5].

Therefore, PC can be modeled as a noncooperative game when each user interacts with other users to maximize its own utility function. This game settles at Nash equilibrium if one exists. However, the Nash equilibrium is not an efficient equilibrium point because of the selfish decisions by each user.

In [2] it is shown that the Nash equilibrium point for PC game is not a Pareto efficient solution for PC in data networks in spite of wireless telephone networks i.e. there are other solutions that produce higher utility for at least one user, without decreasing of any other user utility. Specifically, the uplink power control for a single cell CDMA system is examined and it is shown that if all terminals reduce their powers by a same factor, all terminals achieve higher utility.

The mechanism that proposed in [2] to reduce the terminal powers is called pricing. In a PC game with pricing each terminal decides on its power level to maximize the difference of its utility and the current price which is broadcasted in the network. In fact pricing is used to guide users' behavior toward a more efficient equilibrium point.

This idea is matured in [6], where a distributed algorithm that guarantees the convergence to Pareto dominant equilibrium point is proposed based on supermodular game theorems. Specifically, a pricing function which is a linear function of the transmit power is considered. Each terminal runs the Terminal Algorithm and maximizes the difference between the utility and pricing function in the modified strategy space, while the price is updated at the base station by the Network Algorithm 
and announce to all terminals.

Designing the rules of the game is proposed in [7] as an alternative method to motive individual users to adapt a social behavior and enhance the system performance.

In this paper, we propose a new pricing mechanism for the power control game. In the proposed game, the pricing function is a linear function of the current SINR instead of being a linear function of power suggested in [6]. We first prove that the new game is a supermodular game and then we show that the strategy space of the new game is extended relative to the one in [6]. Therefore, we can expect of achieving a better equilibrium point in running the game with the new proposed pricing function. Simulation results show that the new game can reach a better equilibrium point. Another interesting feature of this new pricing function is that it considers users channel condition in the pricing function. This restricts terminals with bad channel condition to interfere too much for others.

The rest of the paper is organized as follows. In section II, we describe the system model and problem statement in the framework of noncooperative game theory. In section III the required theorems of supermodular game theory is presented and the linear pricing function of [6] is stated. The proposed pricing function is presented in section IV and it is proved that the new game is also supermodular in the strategy space that is computed analytically. Simulation results of both pricing function and discussion on the advantages of the new pricing function is presented in section $\mathrm{V}$.

\section{SYSTEM MOdEL AND BACKGROUND}

Consider a single-cell CDMA system with $N$ stationary users. The path gain from user $j$ to the base station is denoted by $h_{j} . h_{j}$ depends on the user's distance to the base station, $d_{j}$ and the path loss model. Considering a simple propagation model with propagation exponent $4, h_{j}$ is given by $h_{j}=\frac{K_{1}}{d_{j}^{4}}$, where $K_{1}$ is a constant.

The SINR of user $j$ is given by:

$$
\begin{aligned}
\gamma_{j} & =\frac{W}{R} \frac{h_{j} p_{j}}{\sum_{i \neq j} p_{i} h_{i}+\sigma_{j}^{2}}=\frac{p_{j}}{R_{j}} \\
R_{j} & =\frac{\sum_{i \neq j} p_{i} h_{i}+\sigma_{j}^{2}}{\frac{W}{R} h_{j}}
\end{aligned}
$$

Where $W$ is the available spread-spectrum bandwidth [Hz], $R$ is the bit rate $[\mathrm{b} / \mathrm{s}], \sigma_{j}^{2}$ is the AWGN power at the receiver of terminal $j[\mathrm{~W}]$ and $\left(p_{1}, \ldots, p_{N}\right)$ is the power vector of users [W]. $R_{j}$ is effective interference at the base station for user $j$.

The utility function of each user assuming non-coherent FSK modulation is denoted by $u_{j}$ and is given by [2]:

$$
u_{j}=K_{2} \frac{\left(1-e^{-.5 \gamma_{j}}\right)^{M}}{p_{j}}
$$

Where $M$ is the total number of bits per packet and $K_{2}$ is a system dependent constant. As given by (1),(2) the utility function of each user is dependent on its power $p_{j}$ and the power vector of the other users $\mathbf{p}_{-j}$.

When the PC problem is formulated as a noncooperative power control game (NPG), each user maximizes its own utility in a distributed fashion. This game is expressed as [6]:

$$
\begin{gathered}
\max u_{j}\left(p_{j}, \mathbf{p}_{-j}\right) \quad \text { forall } j \in N \\
p_{j} \in P_{j}
\end{gathered}
$$

where $P_{j}=\left[\begin{array}{ll}0 & p_{\max }\end{array}\right]$ is the strategy space of user $j$ and $p_{\max }$ is the maximum allowable power at the terminal transmitter.

NPG has unique Nash equilibrium point and at this point all terminals reach a common target SINR, $\tilde{\gamma}$ that depends on system parameters [6]. This solution is similar to the solution of distributed SINR balancing algorithms that is used in telephone networks [3].

\section{Pricing And Supermodular Games}

The Nash equilibrium point of NPG is inefficient because of the self-optimizing behavior of the users that can degrade the performance of the system. This inefficiency of Nash equilibrium point for power control game has proved in [2], where it is shown analytically that if all users of the system decrease their power by a factor, the utility of all terminals will be increased.

Pricing is a tool that is used to encourage the users to use system resources more efficiently. In an efficient pricing mechanism, decentralized decisions are compatible with the overall system performance. The noncooperative power control game with pricing (NPGP) is formulated as [6]:

$$
\begin{array}{r}
\max u_{j}^{c}\left(p_{j}, \mathbf{p}_{-j}\right)=u_{j}(p)-c_{j}\left(p_{j}, \mathbf{p}_{-j}\right) \\
p_{j} \in P_{j} \quad \text { for all } j \in N
\end{array}
$$

Where $c_{j}: \mathbf{p} \mapsto \mathbb{R}^{+}$is the pricing function of user $j$ and for linear pricing, we have $c_{j}\left(p_{j}, \mathbf{p}_{-j}\right)=c p_{j}$.

The main problem about this new game is about the existence of equilibrium point. The new utility function $u_{j}$ is not concave in $p_{j}$. Therefore, the theorem, which is used for convergence of the NPG, cannot be used here. The supermodular game theory is the tool that is proposed in [6] for the analysis of this game. We briefly express some definitions and main theorems, which are required in the analysis of the new proposed pricing function in the next section.

Lemma 1: If $f$ is twice continuously differentiable, then $f$ has increasing differences in $(x, t)$ if and only if $\frac{\partial^{2} f}{\partial x \partial t}>0$ for all $x, t$

If $f$ has increasing differences, then the set of maximizers $x(t)$ is increasing in $t$ in the sense that both the highest and lowest maximizers will not decrease if $t$ increases. This result is known as Topkis's Theorem [8]. Payoff functions in a supermodular game must have increasing differences in $\left(s_{i}, s_{-i}\right)$ where $s_{i}$ and $s_{-i}$ represent the action of user $i$ and other users.

Definition 1: The game $\left(S_{1}, S_{2}, \ldots, S_{M} ; u_{1}, u_{2}, \ldots, u_{M}\right)$ is a supermodular game if for all $i$ : (1) $S_{i}$ is a compact subset 
of $\mathbb{R}$, (2) $u_{j}$ is upper semi-continuous in $s_{i}, s_{-i}$, (3) $u_{i}$ has increasing differences in $\left(s_{i}, s_{-i}\right)$ [8].

In this definition $S_{i}$ is the strategy space and $u_{i}$ is the payoff function of user $i$. According to this definition, in a supermodular game each player's best response function is increasing in the actions of other players.

Theorem 1: The set of Nash equilibria of a supermodular game is nonempty and has a greatest and least element [8].

Supermodular games have some useful properties. In a class of these games, there is a parameter in the payoff functions that none of the users have control over e.g. in NPGP the price that is broadcasted by the base station is a parameter that affect all utilities and no user has control over it.

Definition 2: A supermodular game is indexed by $t$ if each player payoff function is indexed by $t \in T$, some ordered set, and for all $i, u_{i}\left(s_{i}, s_{-i}, t\right)$ has increasing differences in $\left(s_{i}, t\right)$ [8].

The Nash equilibrium points of these games have a useful property given by theorem 2 .

Theorem 2: If $G$ is a supermodular game indexed by $t$, the largest and smallest Nash equilibria are increasing in $t$ [8].

This theorem shows how the equilibria of such a game shifts when a parameter affect the marginal returns to actions.

The main result of [6] is the following theorem.

Theorem 3: The NPGP is a supermodular game over the strategy space $\left[\begin{array}{ll}p_{j \min } & p_{\max }\end{array}\right]$, where $p_{j \min }$ is computed such that $\gamma_{j \min }>2 \ln M$.

This result is derived by applying definitions (1) and (2) to the game and satisfying the sufficient conditions. Based on theorem (3), two algorithms are proposed in [6], which should run in terminals and base station to reach the new equilibrium in the NPGP.

In the Terminal Algorithm, each terminal computes and applies the minimum power in the modified strategy space i.e. $\gamma_{j \min }>2 \ln M$, that maximized its priced utility function with the price that is broadcasted by the base station. In the Network Algorithm, the base station gets the current utility values for all $j$ at the equilibrium with the most recent advertised price. Then the base station checks to see if all utilities are increased compared to the previous advertised price. If the result is true, the network algorithm increments the price $c:=c+\Delta c$ and announces this price to all terminals.

The algorithm terminates when one terminal experiences a decrease in its utility function due to the increase in the pricing factor. The final price value is called $c_{b e s t}$. As the results of [6] shows, this pricing mechanism has a significant improvement in increasing the network utility and decreasing the power level of all terminals.

Experiments show that the terminal with the worst path gain is the first terminal that experiences the decrease in the utility function. In other words at the termination of the algorithm, the terminal that is farthest to the base station reach its minimum SINR, $\gamma_{\min }=2 \ln M$. Further improvement in the network utility is possible beyond $c_{b e s t}$. However, the supermodularity and existence of the equilibrium point is not guaranteed. Our motivation for the new pricing function is to overcome this restriction such that the game remains supermodular and the strategy space of the game is extended and a lower required bound for SINR is resulted.

\section{The Proposed Pricing Function}

The idea of the new pricing function is a pricing function, which is based on current SINR of each user instead of the current power of user. This pricing function can consider each terminal channel condition implicitly. Therefore, the proposed pricing function has the form $c_{j}\left(p_{j}, \mathbf{p}_{-j}\right)=c \gamma_{j}$.

Proposition 1: The NPGP with the new pricing function is a supermodular game over the strategy space $\left[\begin{array}{ll}p_{j \min } & p_{\max }\end{array}\right]$, where $p_{j \min }$ is computed such that $\gamma_{j \min }>-2 \ln \left(\frac{1}{M}+\frac{c R_{j}}{K_{3} M}\right)$, and $K_{3}$ is a constant related to the constant $K_{2}$ in (2) by $K_{3}=\frac{K_{2} M e^{-1}}{4(M-1)}$.

Proof: We should show that the new strategy space is a sufficient condition for the game to be supermodular according to the definitions (1) and (2). First, we check if the new utility function $u_{j}\left(p_{j}, \mathbf{p}_{-j}, c\right)$ has the increasing differences property in $\left(p_{j}, c\right)$. Changing the variable $t=-c$, to match the definition (2), and taking derivative we have $\frac{\partial^{2} u_{j}}{\partial p_{j} \partial t}=\frac{1}{R_{j}}>0$ for all $j$. We now find the sufficient condition for the new utility function to have the increasing differences in $\left(p_{j}, p_{i}\right)$ for all $i \neq j$. Taking derivative and simplifying the result we have:

$\frac{\partial^{2} u_{j}}{\partial p_{j} \partial p_{i}}=\frac{K_{2} M}{4 R_{j}}\left(1-e^{-.5 \gamma_{j}}\right)^{M-2} e^{-.5 \gamma_{j}}\left[1-M e^{-.5 \gamma_{j}}\right]+c>0$

Note that the condition $\gamma_{j \text { min }}>2 \ln M$, resulted if the expression inside the bracket is greater that zero, is an upper bound solution for this inequality and the inequality is hold for smaller $\gamma_{j \text { min }}$ because of the positive constant c. To find a closed form solution for this inequality, we note that at the boundary, the first term will be negative, therefore at worst case we can substitute $\gamma_{j \text { min }}$ in the term $\left(1-e^{-.5 \gamma_{j}}\right)^{M-2} e^{-.5 \gamma_{j}}$ by $2 \ln (M-1)$ because this term is increasing in $\gamma_{j} \in[02 \ln (M-1)]$. Substituting $\gamma_{j}=$ $2 \ln (M-1)$ we obtain $\left(1-e^{-.5 \gamma_{j}}\right)^{M-2} e^{-.5 \gamma_{j}}<\frac{e^{-1}}{M-1}$. Therefore, it is sufficient to solve the inequality

$$
\frac{K_{3}}{R_{j}}\left[1-M e^{-.5 \gamma_{j}}\right]+c>0, K_{3}=\frac{K_{2} M e^{-1}}{4(M-1)}
$$

This yields the condition

$$
\gamma_{j \min }>-2 \ln \left(\frac{1}{M}+\frac{c R_{j}}{K_{3} M}\right)
$$

Therefore, the minimum required SINR for each terminal decreases for the proposed pricing function and we can hope 
TABLE I

PARAMETER VALUES USED In Simulation

\begin{tabular}{|c||c|}
\hline$M$, number of bits per frame & 80 \\
\hline$W$, spread spectrum bandwidth & $10^{6} \mathrm{~Hz}$ \\
\hline$R$, bit rate & $10^{4} \mathrm{bit} / \mathrm{sec}$ \\
\hline$\sigma^{2}$, AWGN power at the receiver & $5 \times 10^{-15}$ Watt \\
\hline$K_{1}$ & .097 \\
\hline$K_{2}$ & 8000 \\
\hline
\end{tabular}

to reach better equilibrium point. In addition, the minimum required SINR for each terminal is different and depends on the effective interference at the receiver of terminal that can be calculated easily.

The larger the effective interference of a terminal, the lower is the minimum required SINR in the game. Therefore, this pricing function considers terminal channel condition in its pricing function. Terminals with good channel condition encounter lower prices. This is consistent with the opportunistic idea which express that the network efficiency and throughput is improved when terminals with bad channel conditions receives smaller SINR [9].

Another interesting point that we find in simulations is that the new pricing function encourages farther terminals to reduce their power more rapidly compared to the closer ones. The reason is that decreasing in the denominator of (1) is greater for these terminals in two consecutive pricing values when all terminals reduce their powers. This speeds up the convergence of the Network Algorithm with less iteration on updating pricing value $c$.

\section{Simulation Results}

We consider a single cell CDMA system with $N=9$ stationary users. The distance of the terminals to base station is given by $d=$ [200 3004005007008009001000 1100]meters.

Table I summarized other simulation parameters like the setup that is used in [2].

Fig. 1 and Fig. 2 show the utility and power of terminals at equilibrium for NPG, NPGP with linear pricing based on power and NPGP with linear pricing based on SINR. These simulations show an improvement in utility and power with the proposed pricing function. Running NPG, the final SINR of all terminals are the same and equal to 12.24 . Table 2 shows the SINR of terminals at the end of the network algorithm. This table shows that the NPGP with linear pricing based on power terminates where the terminal \#9 reach the minimum allowable SINR of $2 \ln 80=8.76$. However, the NPGP with linear pricing based on SINR passes this point and at the end of the algorithm the SINR of the last three terminals is smaller than 8.76. Therefore, it can reach a better equilibrium point by restricting the minimum required SINR for terminals with bad channel conditions.

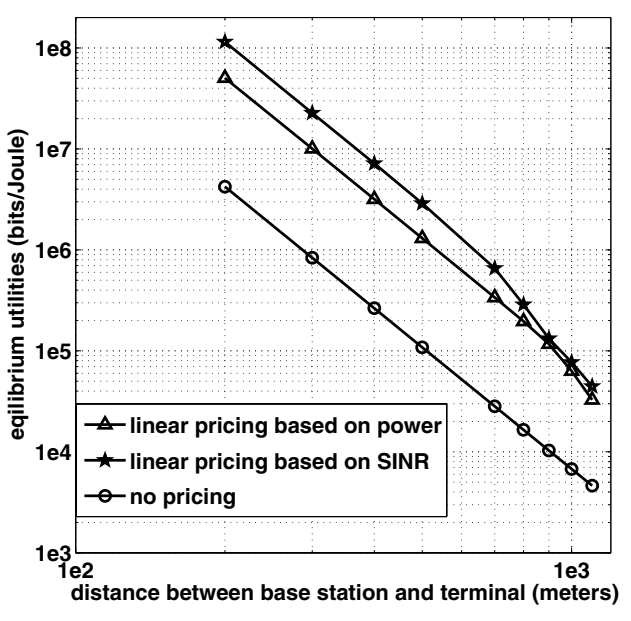

Fig. 1. Terminals utility at equilibrium for NPG, NPGP with power pricing function and NPGP with SINR pricing function

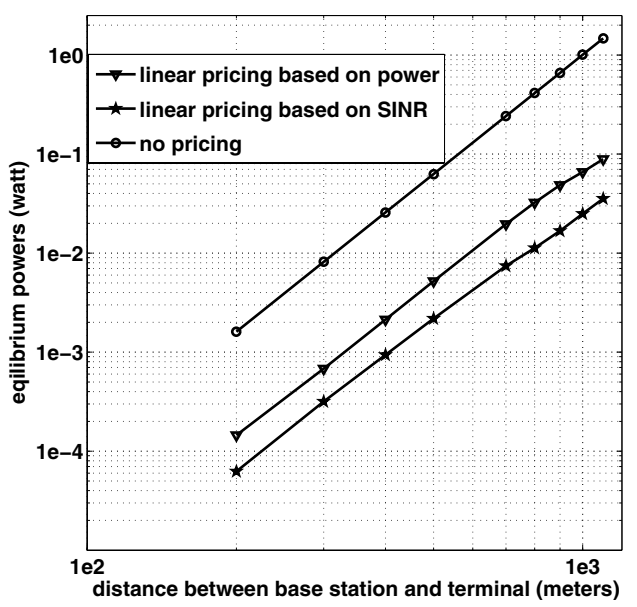

Fig. 2. Terminals power at equilibrium for NPG, NPGP with power pricing function and NPGP with SINR pricing function

TABLE II

FINAL SINR OF TERMINALS

\begin{tabular}{|c||c||c|}
\hline $\begin{array}{c}\text { Terminal } \\
\#\end{array}$ & $\begin{array}{c}\text { Final SINR } \\
\text { with power pricing }\end{array}$ & $\begin{array}{c}\text { Final SINR } \\
\text { with proposed pricing }\end{array}$ \\
\hline 1 & 13.6 & 13.2 \\
\hline 2 & 12.5 & 13.2 \\
\hline 3 & 12.4 & 12.3 \\
\hline 4 & 12.4 & 11.6 \\
\hline 5 & 12.1 & 10.2 \\
\hline 6 & 11.7 & 8.9 \\
\hline 7 & 10.9 & 8.3 \\
\hline 8 & 9.5 & 8.1 \\
\hline 9 & 8.7 & 7.8 \\
\hline
\end{tabular}




\section{CONCLUSION}

Resource allocation problems like PC can be modeled as a noncooperative game. In these games, each terminal interacts with other to achieve its own goal. Usually this behavior of terminals for maximizing their own utility yields an inefficient equilibrium point. Pricing is a mechanism that is used to encourage terminals to use resources more efficiently. This paper proposed a linear pricing function based on each terminal SINR that can achieve this goal. Simulation results of this pricing function compared to linear pricing based on each terminal's power, show an improvement in utility and power consumption of the system.

\section{REFERENCES}

[1] D. Tse and S. Hanly, "Multi-Access Fading Channels: Part I: Polymatroid Structure, Optimal Resource Allocation and Throughput Capacities,"IEEE Trans. on Information Theory, vol. 44, no. 7, pp. 2796-2815, Nov. 1998.

[2] D. J. Goodman, N. B. Mandayam, "Power Control for Wireless Data," IEEE Personal Communications, vol. 7, No. 2, pp. 48-54, April 2000.

[3] G. J. Foschini, Z. Miljanic, "A simple distributed autonomous power control algorithm and its convergence," IEEE Trans. on Vehicular Technology, vol. 42, Issue 4, pp. 641 - 646, Nov. 1993.

[4] R. Yates,"A framework for uplink power control in cellular radio systems," IEEE J. Select. Areas in Commun., vol. 13, no. 7,pp. 1341-1347, Sep. 1995.

[5] A. B. MacKenzie and S. B. Wicker,"Game Theory in Communications: Motivation, Explanation, and Application to Power Control," GlobeCom2001, San Antonio, Texas, November 2001.

[6] C. U. Saraydar, B. Mandayam, D. J. Goodman,"Efficient Power Control via Pricing in Wireless Data Networks,"IEEE Trans. Communications, vol. 50, No. 2, pp. 291-303, February 2002.

[7] Z. Han and K. J. Ray Liu,'Non-Cooperative Power Control Game and Throughput Game over Wireless Networks,"IEEE Trans. Communications, vol.53, no. 10 , Oct. 2005.

[8] J. Levin, Supermodular Games, Notes online available: http://www.eecs.umich.edu/ soniv/research.d/SupermodularGames.pdf, 2003

[9] C. W. Sung and K. K. Leung,"A generalized framework for distributed power control in wireless networks,"IEEE Trans. on Information Theory ,vol. 51, no. 7, pp. 2625-2635, Jul. 2005. 